\section{LIM-only protein FHL2 attenuates vascular tissue factor activity, inhibits thrombus formation in mice and FHL2 genetic variation associates with human venous thrombosis}

\author{
Chantal Kroone, ${ }^{1}$ Mariska Vos, ${ }^{2}$ Timo Rademakers, ${ }^{3}$ Marijke Kuijpers, ${ }^{4}$ \\ Mark Hoogenboezem, ${ }^{3}$ Jaap van Buul, ${ }^{3}$ Johan W.M. Heemskerk, ${ }^{4}$ \\ Wolfram Ruf, ${ }^{5,6}$ Astrid van Hylckama Vlieg, ${ }^{7}$ Henri H. Versteeg, ${ }^{1}$ \\ Marie-José Goumans, ${ }^{8}$ Carlie J.M. de Vries, ${ }^{2 *}$ and Kondababu Kurakula; ${ }^{2,8 *}$ \\ INVENT Consortium
}

${ }^{1}$ The Einthoven Laboratory for Experimental Vascular Medicine, Leiden University Medical Center (UMC), Leiden, the Netherlands; ${ }^{2}$ Department of Medical Biochemistry, Amsterdam Cardiovascular Sciences, Amsterdam UMC, University of Amsterdam, Amsterdam, the Netherlands; ${ }^{3}$ Department of Molecular Cell Biology, Sanquin Research, Amsterdam, the Netherlands; ${ }^{4}$ Department of Biochemistry, Cardiovascular Research Institute Maastricht (CARIM), Maastricht UMC, Maastricht, The Netherlands;

${ }^{5}$ Department of Immunology and Microbiology, The Scripps Research Institute, La Jolla, CA, USA; ${ }^{6}$ Center for Thrombosis and Hemostasis Mainz, Germany; ${ }^{7}$ Department of Clinical Epidemiology, Leiden UMC, Leiden, the Netherlands and ${ }^{8}$ Department of Cell and Chemical Biology, Leiden University Medical Center, Leiden, the Netherlands

${ }^{"} \mathrm{CM} d V$ and KK contributed equally as co-senior authors.

\section{ABSTRACT}

$\mathrm{B}$ leeding disorders and thrombotic complications are major causes of morbidity and mortality with many cases being unexplained. Thrombus formation involves aberrant expression and activation of tissue factor $(\mathrm{TF})$ in vascular endothelial and smooth muscle cells. Here, we sought to identify factors that modulate TF gene expression and activity in these vascular cells. The LIM-only protein FHL2 is a scaffolding protein that modulates signal transduction pathways with crucial functions in endothelial and smooth muscle cells. However, the role of FHL2 in TF regulation and thrombosis remains unexplored. Using a murine model of venous thrombosis in mesenteric vessels, we demonstrated that FHL2 deficiency results in exacerbated thrombus formation. Gain- and loss-of-function experiments revealed that FHL2 represses TF expression in endothelial and smooth muscle cells through inhibition of the transcription factors nuclear factor $\kappa \mathrm{B}$ and activating protein-1. Furthermore, we observed that FHL2 interacts with the cytoplasmic tail of TF. In line with our in vivo observations, FHL2 decreases TF activity in endothelial and smooth muscle cells whereas FHL2 knockdown or deficiency results in enhanced TF activity. Finally, the FHL2 single nucleotide polymorphism rs 4851770 was associated with the risk of venous thrombosis in a large population of venous thrombosis cases and control subjects from 12 studies (INVENT consortium). Altogether, our results highlight functional involvement of FHL2 in TF-mediated coagulation and identify FHL2 as a novel gene associated with venous thrombosis in humans.

\section{Introduction}

Thrombosis is a common pathology underlying venous thromboembolism (VTE), as well as ischemic heart disease and ischemic stroke, and is a leading cause of morbidity and mortality worldwide. ${ }^{1}$ Thrombus formation involves platelet activation and aggregation as well as local, vascular tissue factor (TF) expression and activation, which may result in occlusion of blood vessels and ischemic events. ${ }^{2 \cdot 6}$ The expression of TF, a transmembrane protein, is highly induced in both vascular smooth muscle cells (SMC) and endothelial cells (EC) in response to

Ferrata Storti Foundation

Haematologica 2020
Volume 105(6):1677-1685

\section{Correspondence:}

KONDABABU KURAKULA

k.b.kurakula@lumc.nl

Received: July 25, 2018.

Accepted: August 26, 2019.

Pre-published: August 29, 2019.

doi:10.3324/haematol.2018.203026

Check the online version for the most updated information on this article, online supplements, and information on authorship \& disclosures: www.haematologica.org/content/105/6/1677

(C)2020 Ferrata Storti Foundation

Material published in Haematologica is covered by copyright. All rights are reserved to the Ferrata Storti Foundation. Use of published material is allowed under the following terms and conditions:

https://creativecommons.org/licenses/by-nc/4.0/legalcode. Copies of published material are allowed for personal or internal use. Sharing published material for non-commercial purposes is subject to the following conditions:

https://creativecommons. org//icenses/by-nc/4.0/legalcode, sect. 3. Reproducing and sharing published material for commercial purposes is not allowed without permission in writing from the publisher. 
vascular injury. ${ }^{7-10}$ Upon injury to the vessel wall, TF is exposed to blood coagulation factors. The TF-factor VIIa complex catalyzes the proteolytic activation of coagulation factor $\mathrm{X}$, leading to generation of the multi-purpose enzyme thrombin, which converts fibrinogen into fibrin, activates platelets, induces thrombus formation, and initiates protease-activated receptor (PAR) signaling. ${ }^{11,12}$ It has been demonstrated that TF expression is induced on vascular cells such as EC and SMC as well as on immune cells such as monocytes and may play a pivotal role in a variety of pathological conditions, including acute coronary syndromes, thrombosis, sickle cell disease, diabetes, anti-phospholipid antibody syndrome, septic shock, and cancer. $^{2,4,13-20}$ Furthermore, TF is detectable in macrophages, pericytes and adventitial fibroblasts of normal arteries. ${ }^{21}$

Inflammatory mediators such as tumor necrosis factor$\alpha$ (TNF- $\alpha)$ and pro-thrombotic factors promoting thrombus formation (for example thrombin) have been shown to increase TF expression in vascular cells including EC and SMC. ${ }^{22-24}$ The regulation of TF transcription in EC and SMC, and circulating cells has been described extensively and involves numerous transcription factors such as activating protein-1 (AP-1) and nuclear factor- $\kappa \mathrm{B}(\mathrm{NF} \kappa \mathrm{B}){ }^{25,26}$ In order to identify individuals at risk of thrombosis and to design innovative therapeutic strategies inhibiting thrombus formation in the above-mentioned pathological conditions, it is crucial to identify key factors regulating TF expression and activity in EC and SMC.

LIM-only protein FHL2 is a member of the four and a half LIM (FHL) protein family and is composed of an Nterminal half LIM domain followed by four complete LIM domains. ${ }^{27-31}$ LIM domains contain double zinc finger structures that mediate protein-protein interactions and, unlike other zinc finger structures, show no affinity for DNA. Rather, FHL2 has been shown to interact with a plethora of proteins including nuclear receptors such as Nur77, liver X receptors, androgen receptor, estrogen receptor, and other transcription factors such as AP-1 and NFKB ${ }^{27-31}$ FHL2 is a multifunctional protein and acts as a transcriptional coactivator or corepressor in a cell- and context-dependent manner. Cumulative evidence shows that FHL2 is implicated in a range of physiological and pathological processes, such as cell proliferation, differentiation, migration, and apoptosis, bone formation, wound healing and inflammation. ${ }^{27-31}$ FHL2 is highly expressed in vascular cells including EC and SMC, ${ }^{28-31}$ which is relevant for the current study.

In this study, we investigated the impact of FHL2 on venous thrombosis using ferric chloride $\left(\mathrm{FeCl}_{3}\right)$-induced vascular injury of murine mesenteric vessels. We also demonstrated that FHL2 inhibits TF expression and activity in EC and SMC. Insight into the molecular mechanisms governing this regulation involves evidence that FHL2 regulates TF gene expression in an AP-1- and NFkBdependent manner. Furthermore, we found that FHL2 physically interacts with TF, together modulating local thrombus formation in mice in response to vascular injury. Finally, we identified that the single nucleotide polymorphism (SNP) rs4851770 in the FHL2 gene is associated with venous thrombosis in humans.

\section{Methods}

The methods are described in detail in the Online Supplement.

\section{In vivo thrombosis in mesenteric veins}

Five-week old male FHL2-knockout (KO) mice and respective wildtype (WT) littermate mice (C57BL/6; $\mathrm{n}=8$ per group) were anesthetized by isoflurane ( $2 \%$ for induction, $1.6-1.8 \%$ to maintain anesthesia during imaging) and thrombus formation in the mesenteric veins was provoked as described previously. ${ }^{32}$ Animals were handled in accordance with national and European animal experimental protocols.

\section{Cell culture and transfection}

HEK293T, human umbilical vein endothelial cells (HUVEC), murine and human SMC, and murine macrophages were cultured as described previously. ${ }^{28-30,33,34}$ Human microvascular endothelial cells (HMEC-1) were cultured in MCDB131 medium (Gibco, Blijswijk, the Netherlands) supplemented with $10 \%$ fetal calf serum, epidermal growth factor, penicillin/streptomycin, and Lglutamine. The human endothelial cell line ECRF was cultured in EGM2 medium (Lonza, Basel, Switzerland).

\section{Real-time polymerase chain reaction, western blot, protein stability and co-immunoprecipitation assays}

Real-time polymerase chain reaction analysis, western blot, protein stability and co-immunoprecipitation assays were performed as described previously. ${ }^{28,29}$

\section{FHL2 knockdown}

Recombinant lentiviral particles encoding FHL2 and short hairpin RNA (shRNA) targeting FHL2 were produced, concentrated, and titrated as described previously. ${ }^{24}$

\section{Tissue factor activity and luciferase assays}

TF activity in HUVEC and SMC was assayed as previously described..$^{35}$

Luciferase assays were performed as described previously using TF-promoter luciferase reporter plasmids and full-length FHL2 or FHL2 variants. ${ }^{28,29,36}$

\section{Single nucleotide polymorphism association in patients with venous thromboembolism}

To study the association between FHL2 and VTE, a two-step validation study was designed, consisting of discovery and replication phases. For the discovery phase, 18 FHL2 SNP (listed in Table 1) were tested. In collaboration with the International Network against VENous Thrombosis (INVENT) consortium, the association between these candidate SNP and venous thrombosis was assessed. Details about the design of the INVENT genomewide association study have been previously published. ${ }^{37}$ To obtain replication evidence, SNP rs4851770 $(P<0.000201)$ was selected for genotyping in The Thrombophilia, Hypercoagulability and Environmental Risks in Venous Thromboembolism (THE-VTE) case-control study including 676 patients with a first objectively diagnosed episode of deep venous thrombosis aged $18-70$ years and 368 control subjects. This twocenter, case-control study has been previously described. ${ }^{38}$ Human studies were performed with patients' consent and approval from the institutional review boards of participating research centers and hospitals.

\section{Statistical analysis}

All statistical analyses were carried out with GraphPad Prism software (GraphPad Software, San Diego, CA, USA). Comparisons between two groups were done with the Student $t$ test for unpaired variables. Comparisons between more than two 
groups were testesd by analysis of variance (ANOVA). Data are reported as mean \pm standard deviation. $P$ values $<0.05$ were considered statistically significant.

The $P$ values obtained from the INVENT genome-wide association study were corrected using the Bonferroni adjustment. In the THE-VTE study, the association of the genotyped SNP with the risk of a first venous thrombosis was assessed by calculating odds ratios (OR) with corresponding 95\% confidence intervals $(95 \%$ CI) adjusted for age and sex, using SPSS (SPSS Inc, Chicago, IL, USA). Analyses were performed in the overall group of patients and after stratification into groups with provoked or unprovoked first venous thrombosis. An unprovoked venous thrombosis was defined as an event in the absence of surgery, plaster cast, injury, immobilization for more than four consecutive days, hospitalization, pregnancy or postpartum status, or hormone use in the 3 months prior to the event. ${ }^{37}$

\section{Results}

FHL2-deficient mice show enhanced thrombus formation upon vascular injury

We previously reported that FHL2 deficiency causes enhanced arterial lesion formation in the murine model of carotid artery ligation. ${ }^{30}$ Interestingly, even 4 weeks after ligation, thrombus formation was observed in sections of
A
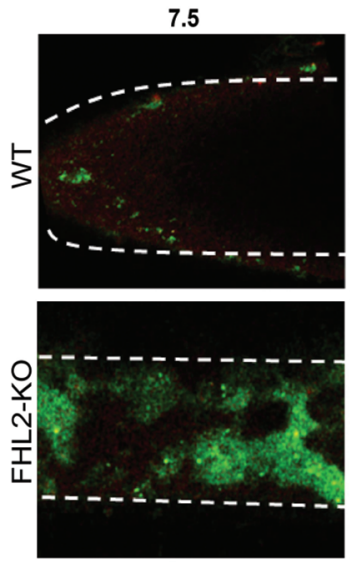

15
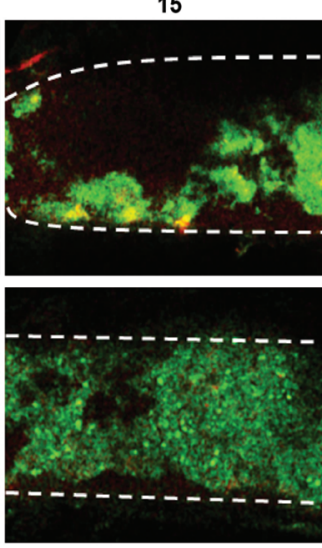

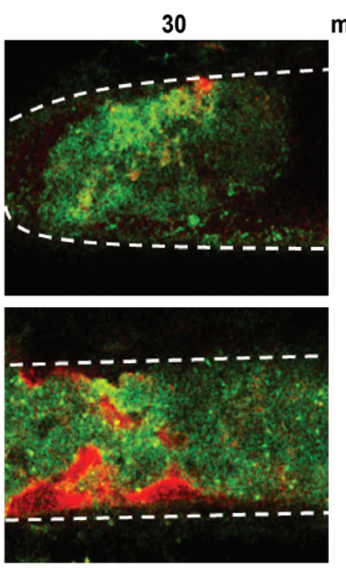

B

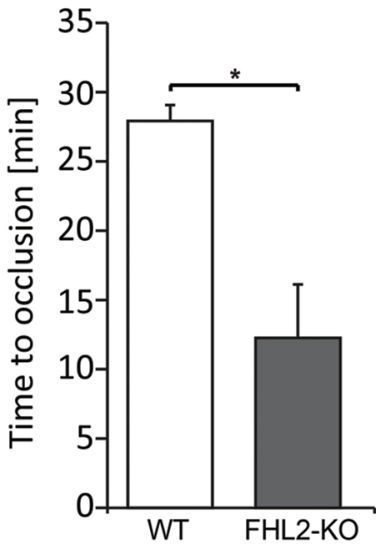

Figure 1. FHL2-deficient mice show enhanced thrombus formation upon vascular injury. Ferric chloride (FeCl ${ }_{3}$ )-induced thrombus formation in wildtype (WT) and FHL2-deficient (FHL2-KO) mice. (A) Representative photomicrographs are shown at different time points after injury in mesenteric vessels of WT and FHL2-KO mice. Platelets were visualized with a fluorescently labeled antibody (green) and labeled fibrinogen was injected to visualize fibrin formation in the thrombus (red). (B) The time to occlusion was assessed, revealing a 2.3-fold faster occlusion in FHL2-KO mice compared to WT animals. Data represent means \pm standard deviation. $* P<0.05 ; \mathrm{n}=8$.

Table 1. FHL2 single nucleotide polymorphism analyses in the INVENT cohort.

\begin{tabular}{|c|c|c|c|c|c|}
\hline $\begin{array}{l}\text { Gene: FHL2 } \\
\text { SNP }\end{array}$ & Chr:Pos & EA/NEA & BETA & SE & $P$ \\
\hline rs11124029 & 2:105977761 & $\mathrm{G} / \mathrm{A}$ & 0.032368 & 0.030102 & 0.282235 \\
\hline rs3087523 & 2:105977776 & $\mathrm{G} / \mathrm{A}$ & -0.022763 & 0.035866 & 0.525667 \\
\hline rs2278501 & 2:105979506 & $\mathrm{T} / \mathrm{C}$ & 0.001005 & 0.023185 & 0.965399 \\
\hline rs2278502 & $2: 105979730$ & $\mathrm{C} / \mathrm{A}$ & 0.024248 & 0.024195 & 0.316255 \\
\hline rs2576778 & $2: 105982753$ & $\mathrm{G} / \mathrm{A}$ & 0.013155 & 0.02822 & 0.641123 \\
\hline rs880427 & $2: 105985228$ & $\mathrm{G} / \mathrm{A}$ & 0.024726 & 0.024537 & 0.313577 \\
\hline rs4640402 & 2:105999009 & $\mathrm{A} / \mathrm{C}$ & 0.011695 & 0.02346 & 0.618169 \\
\hline rs4851765 & 2:106012632 & $\mathrm{T} / \mathrm{C}$ & 0.030595 & 0.024279 & 0.207594 \\
\hline rs11891016 & $2: 106013216$ & $\mathrm{C} / \mathrm{T}$ & 0.031439 & 0.024313 & 0.195951 \\
\hline rs11884297 & $2: 106013248$ & $\mathrm{C} / \mathrm{T}$ & -0.015773 & 0.025252 & 0.532232 \\
\hline rs4374396 & $2: 106024451$ & $\mathrm{~A} / \mathrm{G}$ & 0.009225 & 0.023494 & 0.694594 \\
\hline rs2376740 & 2:106032291 & $C / T$ & -0.056017 & 0.02413 & 0.02028 \\
\hline rs1914748 & $2: 106035580$ & $C / T$ & 0.05547 & 0.023114 & 0.016425 \\
\hline rs4851770 & 2:106046333 & $\mathrm{C} / \mathrm{T}$ & -0.086598 & 0.023279 & 0.000201 \\
\hline rs6750100 & $2: 106046789$ & $A / G$ & 0.056783 & 0.02598 & 0.028863 \\
\hline rs4851772 & 2:106051956 & $\mathrm{A} / \mathrm{G}$ & -0.005646 & 0.038529 & 0.883475 \\
\hline rs7583367 & $2: 106053343$ & $\mathrm{G} / \mathrm{T}$ & -0.039482 & 0.023328 & 0.090562 \\
\hline rs10177620 & $2: 106083368$ & $\mathrm{~A} / \mathrm{G}$ & -0.031521 & 0.025241 & 0.211709 \\
\hline
\end{tabular}

SNP: single nucleotide polymorphism. Chr:Pos: chromosome: position; EA_ effect allele; NEA: non-effect allele; SE: standard error SNP with a $P$ value $<0.05$ are shown in italics The SNP that is significantly associated after Bonferroni correction is in bold. 
the ligated arteries in five of 14 FHL2-KO mice, whereas such structures were absent in the vascular lesions of WT mice (data not shown). We therefore hypothesized that FHL2 may be involved in thrombus formation. To substantiate this hypothesis, we performed $\mathrm{FeCl}_{3}$-induced thrombosis experiments in WT and FHL2-KO mice. Upon
$\mathrm{FeCl}_{3}$-induced vascular injury, the blood flow in mesenteric veins was imaged by intravital microscopy to assess thrombus formation. Platelets were visualized with an anti-GPIb $\beta$ antibody and fluorescent fibrinogen was injected to monitor fibrin accumulation (Figure 1A). The time from vessel injury to formation of a stable occlusive

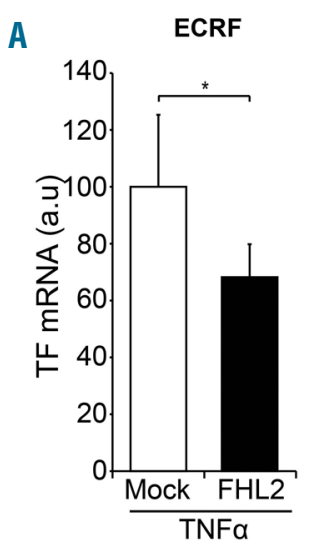

$\mathbf{F}$

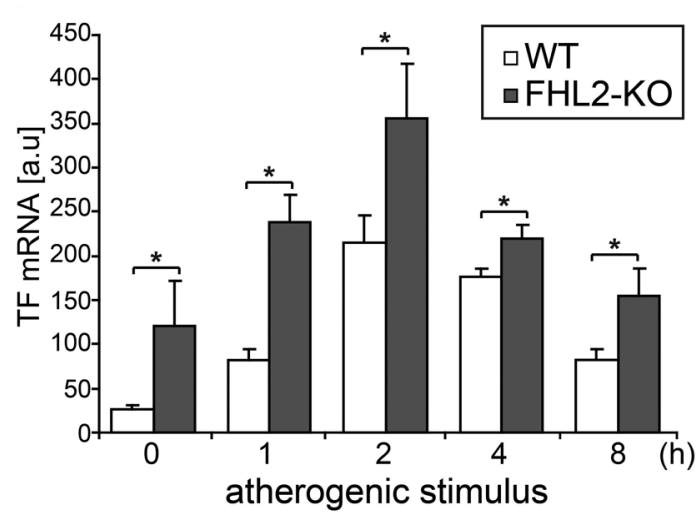

H

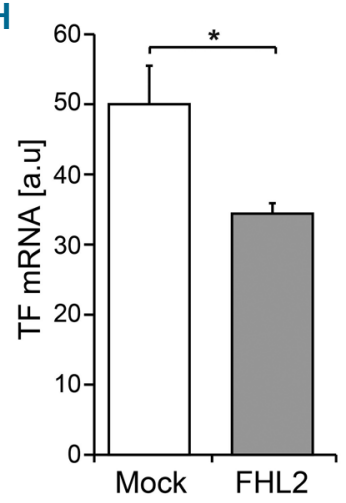

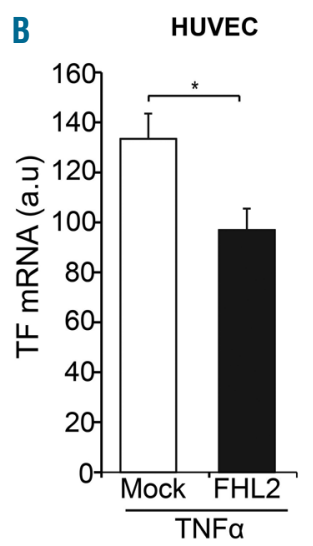

C

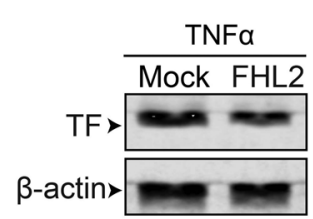

D

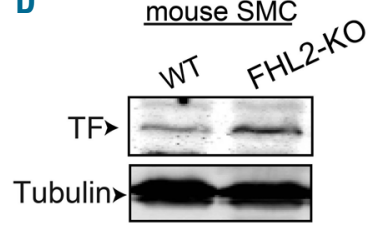

G

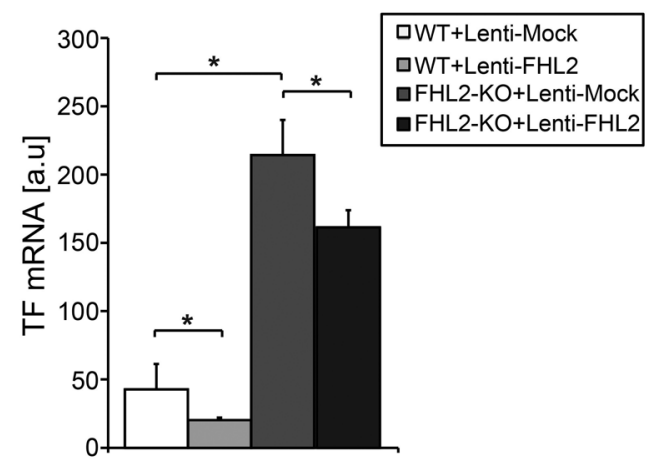

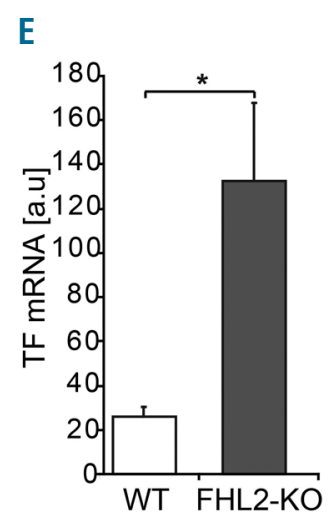

$\mathbf{J}$

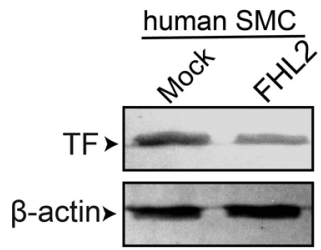

Figure 2. FHL2 modulates tissue factor expression in endothelial cells and smooth muscle cells. (A) Tissue factor (TF) mRNA levels were determined by real-time quantitative polymerase chain reaction (qRT-PCR) in ECRF cells (A) and in human umbilical vein endothelial cells (HUVEC) (B) transduced with control lentivirus (Mock) or FHL2-encoding virus after treatment with tumor necrosis factor- $\alpha$ (TNF $\alpha$ ) (C) Western blot analysis of TF protein expression in HUVEC following ectopic expression of FHL2 and stimulation with TNFa. (D) Western blot analysis of TF in aortic smooth muscle cells (SMC) derived from wildtype (WT) and FHL2-knockout (KO) mice. Tubulin was used as a loading control. (E) To assess mRNA expression of TF in the aortic SMC isolated from WT and FHL2-KO mice, qRT-PCR was performed. (F) TF mRNA levels were determined by qRT-PCR in WT and FHL2-KO SMC stimulated with macrophage-conditioned medium (an atherogenic stimulus, see Methods) for the indicated time periods. (G) WT and FHL2-KO SMC were transduced with lentiviral particles encoding control (Mock) or FHL2 and assayed for TF mRNA expression by qRT-PCR. (H) Gain-of-function and (I) knockdown of FHL2 in human SMC, after which mRNA expression of TF was determined. Data represent means + standard deviation (SD). $* P<0.05$. shCtrl, short-hairpin control. a.u, arbitrary units. (J) Human SMC were transduced with lentiviral particles encoding control (Mock) or FHL2 and western blot analysis was performed to assess TF protein levels. In all qRT-PCR experiments acidic ribosomal phosphoprotein PO was determined as an internal control for cDNA content of the samples and data are represented as means \pm SD. $* P<0.05$. Western blot analyses were performed to assess TF protein levels and $\beta$-actin was used as a loading control. $n=3$ 
A

TF-promoter
\begin{tabular}{|c|c|c|}
\hline NFKB & AP1 & Luc \\
\hline $\mathrm{X}$ & WP 1 & Luc \\
\hline NFKB-mut \\
\hline NFKB & $X$ & Luc \\
\hline
\end{tabular}

B

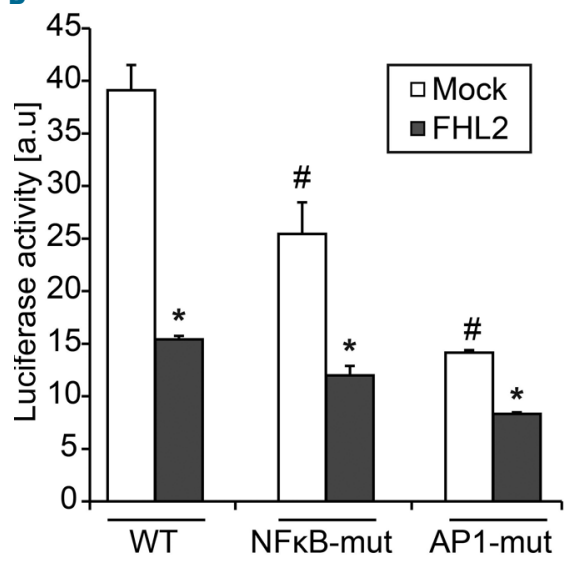

C

\begin{tabular}{|c|c|c|c|c|c|}
\hline FHL2 & 0 & 1 & 2 & 3 & 4 \\
\hline LIMO-1 & 0 & 1 & & & \\
\hline LIMO-2 & 0 & 1 & 2 & & \\
\hline LIMO-3 & 0 & 1 & 2 & 3 & \\
\hline
\end{tabular}

D

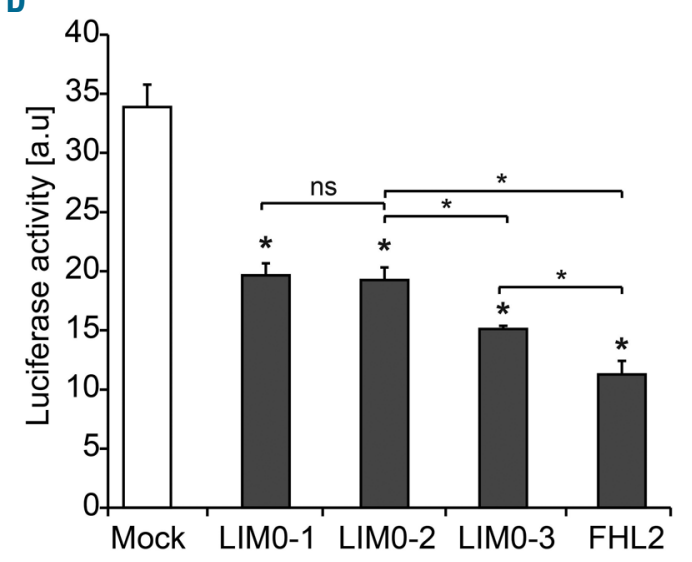

Figure 3. FHL2-mediated regulation of tissue factor promoter luciferase reporter. (A) Schematic representation of the normal (WT) and mutant human tissue factor (TF) promoter-reporter constructs that were used. (B) Transient co-transfection of 293T cells was performed with either TF-Luc (WT), NFKB mut-Luc and AP-1 mut-Luc in combination with either a control plasmid (Mock) or a plasmid encoding FHL2 and luciferase activity was measured after stimulating the cells with phorbol myristate acetate (PMA). (C) Schematic representation of the FHL2 variants that were tested. (D) The effect of FHL2 variants on TF promoter activity was assayed following PMA stimulation in comparison with mock-plasmid transfected cells (Mock). Luciferase activity was normalized to Renilla. Data represent means \pm standard deviation. $* P<0.05$. a.u, arbitrary units. $\mathrm{n}=3$

thrombus (time to occlusion) was 2.3-fold shorter in FHL2-KO mice, suggesting that FHL2 has an inhibitory function in thrombus formation following vascular injury (Figure 1B). Enhanced fibrin formation was observed in vessels of FHL2-KO animals and was visible as intense red fluorescence (Online Supplementary Figure S1). Since $\mathrm{FeCl}_{3}-$ induced thrombus formation involves TF expression and activity in the vessel wall, ${ }^{39}$ we next studied the impact of FHL2 on this aspect in EC and SMC.

\section{FHL2 deficiency enhances tissue factor expression}

Given that TF expression in $\mathrm{SMC}$ is required for $\mathrm{FeCl}_{3}$ induced vascular injury, we hypothesized that FHL2 may have a function in modulating TF expression in EC and SMC. ${ }^{40}$ We therefore determined TF expression in the endothelial cell line ECRF and in HUVEC following overexpression of FHL2. Ectopic expression of FHL2 reduced TNF $\alpha$-induced TF mRNA and protein expression in ECRF cells and HUVEC (Figure 2A-C; Online Supplementary Figure S2A). Similar results were observed in human microvascular endothelial cells (data not shown).

Next, we cultured SMC of WT and FHL2-KO mice and assessed the expression of TF. TF mRNA expression was significantly higher in SMC cultured from FHL2-KO than in those from WT mice (Figure 2D-E; Online Supplementary Figure S2C). The expression of TF in SMC was increased in response to a conditioned medium of macrophages stimulated with oxidized low density lipoproteins, which may be considered as an atherogenic stimulus. ${ }^{33}$ Also under these conditions TF expression was higher in FHL2-
Table 2. Validation of results of the single nucleotide polymorphism rs4851770 in the THE VTE cohort.

\begin{tabular}{lccc} 
& Patients & Controls & OR (95\% Cl)* \\
All & & & \\
TT & $145(21.5 \%)$ & $84(22.5 \%)$ & $1[\mathrm{ref}]$ \\
CT & $340(50.4 \%)$ & $200(53.5 \%)$ & $1.00(0.72-1.38)$ \\
CC & $190(28.1 \%)$ & $90(24.1 \%)$ & $1.21(0.83-1.75)$ \\
Unprovoked & & & \\
TT & $58(17.3 \%)$ & $84(22.5 \%)$ & $1[\mathrm{ref}]$ \\
CT & $180(53.6 \%)$ & $200(53.5 \%)$ & $1.25(0.82-1.89)$ \\
CC & $98(29.2 \%)$ & $90(24.1 \%)$ & $1.53(0.96-2.45)$ \\
\hline
\end{tabular}

OR: odds ratio; $95 \%$ CI: $95 \%$ confidence interval. *OR adjusted for age and sex.

KO SMC than in WT cells (Figure 2F). Subsequently, gainof-function experiments were performed in both WT and FHL2-KO SMC (FHL2 expression is shown in Online Supplementary Figure S2D). FHL2 downregulated TF expression in both WT and FHL2-KO SMC (Figure 2G). Similarly, in human SMC the expression of TF mRNA and protein decreased following ectopic FHL2 expression (Figure 2H, J; Online Supplementary Figure S2B), whereas knockdown of FHL2 in human SMC resulted in enhanced expression of TF mRNA (Figure 2I; FHL2 expression is shown in Online Supplementary Figure S2E). Furthermore, we confirmed increased TF expression in FHL2-KO mice in RNA samples from isolated carotid arteries after carotid artery ligation for 1, 2 and 4 weeks and in intact, isolated 
mouse aorta to rule out confounding tissue culture-related effects (Online Supplementary Figure S3A, B). We also demonstrated that bone-marrow derived macrophages from FHL2-KO mice showed increased mRNA levels of TF under basal, lipopolysaccharide-, and interleukin-4stimulated conditions compared to macrophages derived from WT mice (Online Supplementary Figure S3C). Together, these data indicate that FHL2 inhibits TF expression in EC, SMC and macrophages.
FHL2 regulates tissue factor expression through inhibition of nuclear factor $\kappa \mathrm{B}$ and activating protein-1

FHL2 does not bind DNA directly but is known to affect the activity of genuine transcription factors such as NFKB and AP-1. The TF promoter contains functional binding motifs for NFKB and AP-1. ${ }^{25,26}$ To elucidate the mechanism by which FHL2 suppresses transcription of the TF gene, we performed transient transfections with WT and mutant TF promoter-reporter constructs in HEK293T cells
A

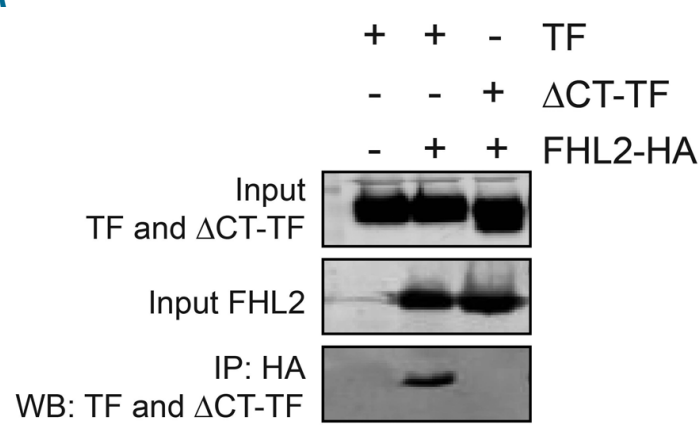

B

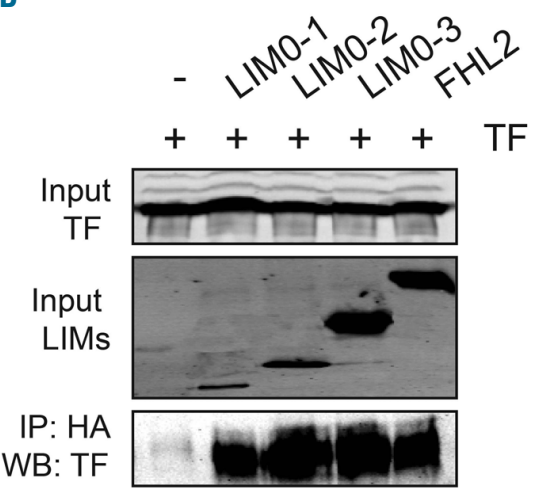

C

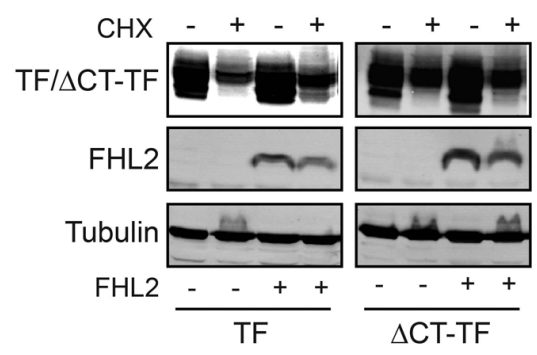

D

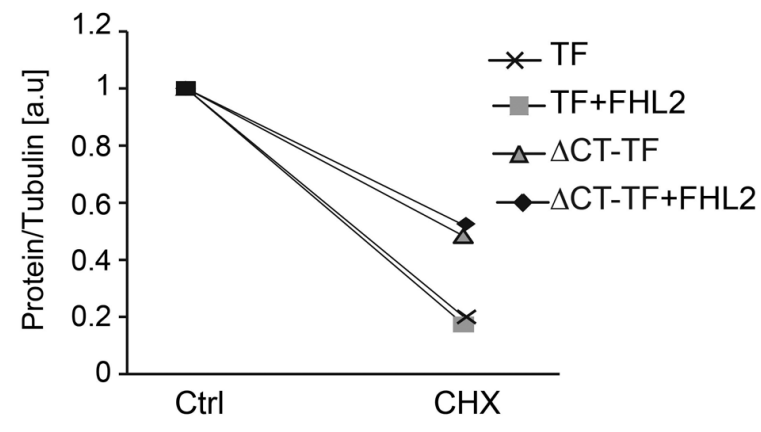

G

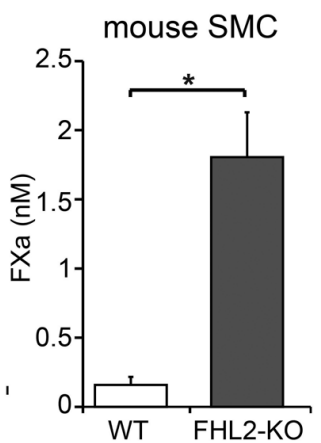

H

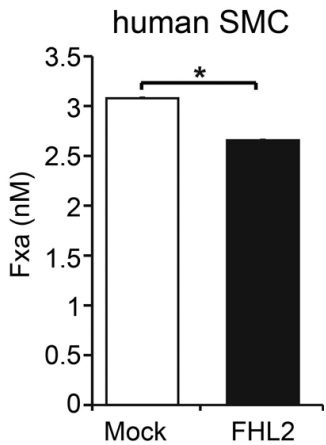

Figure 4. FHL2 physically interacts with tissue factor and inhibits tissue factor pro-coagulant activity. (A) HEK 293T cells were co-transfected with expression vectors encoding HA-tagged FHL2 and full-length tissue factor (TF) or TF lacking the intracellular cytoplasmic TF domain ( $\triangle \mathrm{CT}$-TF), as indicated. Whole cell extracts were immunoprecipitated using the anti-HA antibody (IP: HA) and analyzed by western blotting (WB) with an anti-TF antibody (WB: TF and $\Delta$ CT-TF). Input of TF (Input TF and $\triangle$ CT-TF) and FHL2 (Input FHL2) were revealed on a separate blot. (B) HEK293T cells were co-transfected with expression vectors encoding HA-tagged FHL2 variants and TF, as indicated. After immunoprecipitation with the anti-HA antibody (IP: HA) the samples were analyzed by western blotting with anti-TF antibody (WB: TF). Input samples were probed for FHL2 variants (Input LIMs) and TF (Input TF). (C, D) HEK293T cells were transfected with expression plasmids coding for TF or $\triangle$ CTTF with or without FHL2. Cells were treated with cycloheximide (CHX) to block de novo protein synthesis for $16 \mathrm{~h}$. Tubulin was used as the loading control. Western blot analysis (C) and quantification of western blots (D); a.u, arbitrary units. (E, F) Human umbilical vein endothelial cells (HUVEC) and human micro-vascular endothelial cells (HMEC-1) were transduced with lentivirus encoding control shRNA (shCtrl) or shFHL2 followed by serum-starvation and treatment with tumor necrosis factor- $\alpha$ (TNF $\alpha$ ). The generation of activated factor X (FXa) was measured. (G) FXa generation was assayed in smooth muscle cells (SMC) derived from WT and FHL2 $\mathrm{KO}$ mice following treatment with ionomycin. $(\mathrm{H})$ FXa generation was assayed in human SMC upon ectopic expression of FHL2 or transduced with control lentivirus (Mock) and treatment with TNF $\alpha . \quad n=3$. 
(Figure 3A). FHL2 expression decreased the luciferase activity of the WT TF-promoter reporter (Figure 3B). TF promoter constructs with either a disrupted NFKB response element or a mutated AP-1 element exhibited lower basal luciferase activity (Figure 3B) and FHL2-mediated inhibition of luciferase activity of the WT-promoter $(61 \%)$ was stronger than that of the NFKB- or AP-1-mutated promoter $(52 \%$ and $43 \%$, respectively) (Figure $3 \mathrm{~B}$ ). These data suggest that FHL2 affects the transcriptional activity of both NFKB and AP-1 to downregulate TF-promoter activity in these cells. To assess whether activation of EC affects cellular localization of FHL2, we stimulated EC with TNF- $\alpha$ (50 ng/mL) and observed enhanced nuclear localization, which may contribute to the changed modulation of transcription factor activity by FHL2 (Online Supplementary Figure S4). To determine which domain of FHL2 is essential to inhibit TF-promoter activity, deletion mutants of FHL2 ${ }^{28}$ (Figure 3C) were tested in TF-promoter luciferase assays. The first one and a half LIM-domain (LIM0-1) already exhibited partial inhibition (41\%) with the LIM0-2 variant having similar effects, while LIM0-3 (55\% inhibition) and FHL2 were more potent inhibitors of TF-promoter activity (Figure 3D). Of note, overexpression of individual LIM domains of FHL2 had no effect on TF promoter activity (data not shown) indicating that at least one and a half LIM domains of FHL2 are required to elicit decreased TF-promoter activity.

\section{FHL2 physically interacts with tissue factor}

To investigate whether inhibition of TF by FHL2 is also attributable to this latter's physical interaction with TF, we performed co-immunoprecipitation experiments. Fulllength TF co-immunoprecipitated efficiently with HAtagged FHL2 from whole cell extracts using an anti-HA antibody (Figure 4A), whereas $\Delta$ CT-TF, which lacks the intracellular cytoplasmic TF domain, failed to bind FHL2 (Figure 4A), indicating that FHL2 requires the relatively short cytoplasmic tail of TF for its interaction. Pull-down assays using FHL2 deletion mutants (Figure 3C) revealed that all variants of FHL2 bind TF (Figure 4B). It is known that FHL2 can enhance protein stability and to measure the effect of FHL2 on TF protein stability, we exposed cells to cycloheximide and determined the half-life of fulllength TF and $\Delta$ CT-TF mutant proteins. The $\Delta C T-T F$ variant was more stable than full-length TF, but TF protein levels were not influenced by FHL2 (Figure 4C, D). We concluded that FHL2 interacts with TF but does not affect TF protein stability.

\section{FHL2 modulates tissue factor procoagulant activity}

Having established that FHL2 interacts with TF and regulates TF expression in EC and SMC, we sought to examine the impact of FHL2 on the activity of TF in cultured EC and murine and human SMC, by assessing the potential of these cells to generate factor $\mathrm{Xa}(\mathrm{FXa})$. Knockdown of FHL2 in HUVEC and HMEC-1 resulted in enhanced TNF $\alpha$-induced FXa generation compared to control (Figure 4E, F). In a similar fashion, FHL2-deficient mouse SMC showed higher ionomycin-induced FXa generation than WT SMC (Figure 4G). Ectopic expression of FHL2 resulted in a modest decrease of TNF $\alpha$-induced FXa generation compared to that of control-transduced cells (Figure 4H). Of note, following FHL2 knockdown in HMEC-1, we found, in the same cell lysates, that TF protein levels were increased 1.5-fold and that FXa generation was increased even more, 3-fold (Online Supplementary Figure $S 5 A, B)$. These data indicate that FHL2 may regulate TF activity partly by binding the intracellular domain of TF and by modulating $T F$ gene expression. Figure 5 is a schematic summary of our data.

\section{Genetic variation in the FHL2 gene is associated with venous thrombosis in humans}

Having established that FHL2 reduces TF activity in EC and SMC, we reasoned that genetic variation in FHL2 may affect venous thrombosis in man. We therefore analyzed 18 FHL2 SNP and venous thrombosis risk in the INVENT consortium (Table 1). We observed that the FHL2 polymorphism rs 4851770 was associated with thrombosis risk with a $P$-value of $2 \times 10^{-4}$, even after correction for multiple testing. No association was found between other tested SNP in the FHL2 gene and risk of venous thrombosis
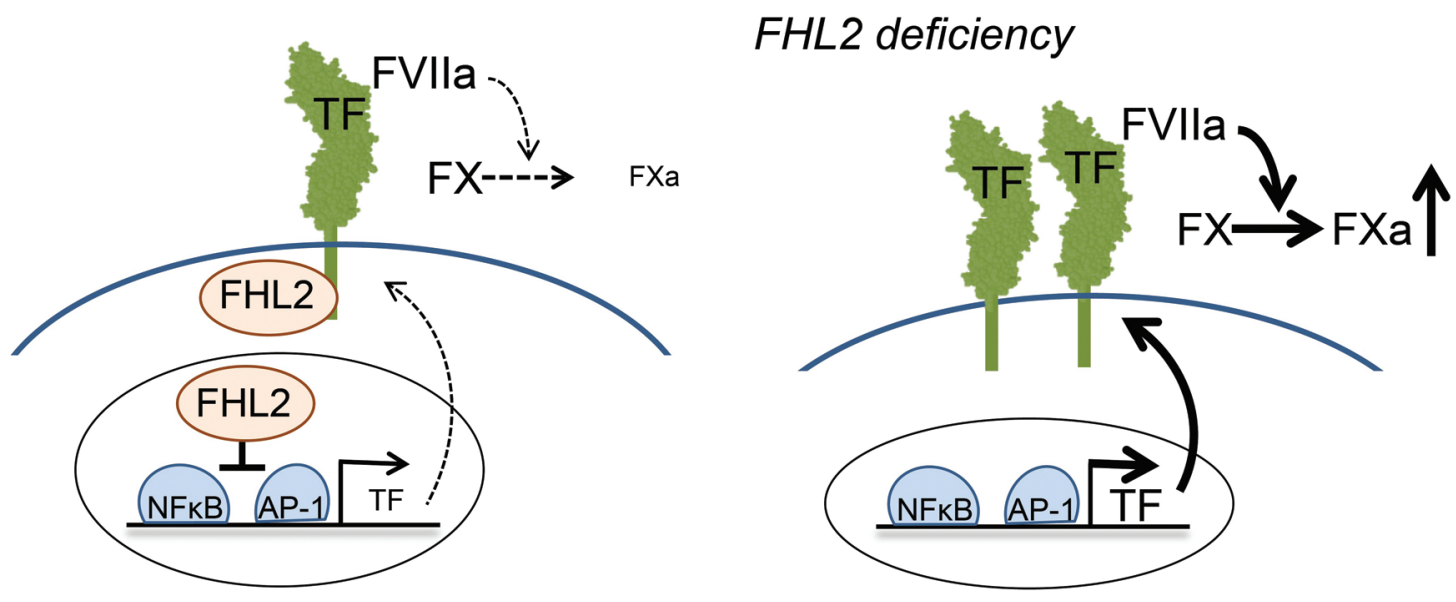

Figure 5. Schematic representation of FHL2 function in the modulation of tissue factor activity. The left panel shows the normal situation in which FHL2 inhibits tissue factor (TF) expression in activated endothelial cells (EC) and smooth muscle cells (SMC), whereas the right panel represents the effect of FHL2 deficiency, which results in enhanced TF expression and increased activity. 
(Table 1). Next, we sought for replication in the THE-VTE study. Individuals homozygous for the rs4851770C Callele had a mildly increased risk of venous thrombosis compared with individuals homozygous for the T-allele (OR: 1.2; 95\% CI: 0.7-1.4). The risk of idiopathic venous thrombosis was more pronounced (OR:1.53 (95\% CI: 0.96 - 2.45) (Table 2).

\section{Discussion}

Thrombosis-associated pathologies, such as VTE, myocardial infarction and stroke, are major causes of morbidity and mortality worldwide. TF is a key player in the extrinsic pathway of coagulation ${ }^{14}$ and although many experimental studies have helped us to understand the coagulation process, the regulation of TF expression and activity is incompletely understood. In the current study, we demonstrated that deficiency of FHL2 exacerbates thrombus formation in response to $\mathrm{FeCl}_{3}$-induced vascular injury. We revealed that FHL2 inhibits the expression of TF in activated vascular cells, including EC and SMC. Furthermore, we showed that FHL2 interacts with the intracellular domain of TF and inhibits TF activity. Additionally, we found that the FHL2 rs4851770 polymorphism is associated with venous thrombosis in humans.

We previously demonstrated that FHL2-KO mice develop larger SMC-rich lesions in the murine carotid artery ligation model and here we observed that these lesions comprised, even 4 weeks after ligation, more thrombi than lesions in WT mice..$^{30}$ The carotid artery ligation model is, however, not suitable for quantitative assessment of changes in venous thrombosis. For that reason, in this study we used the $\mathrm{FeCl}_{3}$-induced vascular injury model and established that FHL2-KO mice do indeed show exacerbated venous thrombus formation compared to WT mice.

SMC are the major source of $\mathrm{TF}$ in the $\mathrm{FeCl}_{3}$-induced model of vascular injury. Although there are some contrasting data on TF expression in EC, many studies demonstrated that TF is highly induced in endothelium under inflammatory conditions. Moreover, enhanced activity of TF is transient and directly correlates with increased mRNA levels. ${ }^{22-24}$ In the current study we found that overexpression of FHL2 inhibits TF expression, whereas FHL2 deficiency results in higher TF levels and activity. These observations were corroborated in HUVEC and microvascular HMEC-1 cells indicating that FHL2mediated regulation of TF is not limited to larger vessels but may also occur in microvessels.

Thrombin is generated after TF exposure and is known to promote vascular neointima formation through multiple mechanisms, including activation of platelets and induction of SMC proliferation. ${ }^{41,42}$ Given that FHL2 inhibits SMC proliferation ${ }^{28,30}$ and our current observation that the level of active TF is increased in SMC deficient in FHL2, we hypothesize the following mechanism: in FHL2-KO mice enhanced TF expression promotes thrombin generation that in turn accelerates SMC proliferation causing enhanced lesion formation in the carotid artery ligation model. In addition to increased TF expression levels, we also observed that FHL2-KO SMC produce more pro-inflammatory cytokines than WT SMC, which may also contribute to the pro-thrombotic phenotype of FHL2$\mathrm{KO}$ mice (data not shown). Further studies are warranted to investigate the hypothesis that thrombin actually mediates the increased SMC-rich lesion formation observed in FHL2-KO mice.

It is well established that the human TF gene contains binding sites for the transcription factors NFKB, AP-1, Sp1 and Egr-1..$^{25,26}$ Interestingly, it has been reported that FHL2 is associated with these four transcription factors in multiple cell types in distinct contexts. ${ }^{29,31,43}$ Here, we demonstrated that FHL2 regulates TF-promoter activity through modulation of both NFKB and AP-1. The relative contribution of FHL2 in regulation of the activity of Egr-1 and Sp1 on the TF promoter is difficult to assess, because deletion of the respective response elements completely abrogates the activity of this promoter, as has been shown before. We found that FHL2 physically interacts with fulllength TF, but not with the cytoplasmic tail-deleted mutant of TF $(\triangle \mathrm{CT}-\mathrm{TF})$ indicating that FHL2 interacts intracellularly at the cell membrane with TF. This finding may relate to the interaction of FHL2 with several integrin units such as $\alpha_{3} \beta_{1}$, which form a complex with TF.44,45 It is not unlikely that FHL2 either stabilizes or abolishes the integrin $\alpha_{3} \beta_{1}$-TF complex, thereby affecting downstream signaling. Further studies are required to investigate the exact role of FHL2 in such TF complexes.

We explored the association between FHL2 polymorphisms (Table 1) and venous thrombosis risk demonstrating that rs 4851770 is associated. FHL2 was not previously known as a direct thrombo-modulator, although it has been shown to modulate the thrombosis-associated genes PAI- ${ }^{46}$ and eNOS. ${ }^{47}$ At present, we can only speculate on the functional implication of the FHL2 polymorphism rs4851770 in venous thrombosis. We postulate that this polymorphism affects the binding of specific transcription factors on the FHL2 promoter, resulting in modulation of FHL2 expression and, as a consequence, regulates TF activity.

In summary, we demonstrated that FHL2 is a novel regulator of TF in vascular EC and SMC. Furthermore, we report that FHL2 regulates TF promoter activity partly through $\mathrm{NF \kappa B}$ and AP-1. Finally, we showed that FHL2 physically interacts with full-length TF. This work reinforces the biological significance of FHL2 as a regulator of venous thrombosis, which may be of significance to identify individuals at risk of VTE, and suggest that enhancement of FHL2 expression may even be a target for intervention.

\section{Acknowledgments}

We thank Kamran Bakhtiari and Joost C.M. Meijers (Dept. of Plasma Proteins, Sanquin Research, Amsterdam, the Netherlands) for assistance with the TF activity assays. We also thank Dr. Nigel Mackman (University of North Carolina) for TF-promoter luciferase reporter plasmids.

\section{Funding}

This work was supported by the research program of the BioMedical Materials Institute, co-funded by the Dutch Ministry of Economic Affairs as a part of Project P1.02 NEXTREAM. This work was also supported by the Rembrandt Institute for Cardiovascular Research (RICS grant 2013), the Netherlands CardioVascular Research Initiative: the Dutch Heart Foundation (CVON: 2012-08) and the Dutch Lung Foundation (5.2.17.198JO). 


\section{References}

1. Raskob GE, Angchaisuksiri P, Blanco AN., et al. Thrombosis: a major contributor to the global disease burden. J Thromb Haemost. 2014:12(10):1580-1590

2. Aras $O$, Shet $A, B a c h R R$, et al. Induction of microparticle- and cell-associated intravascular tissue factor in human endotoxemia. Blood. 2004;103(12):4545-4553.

3. Cimmino G, Ciccarelli G, Golino P. Role of tissue factor in the coagulation network. Semin Thromb Hemost. 2015;41(7):708717.

4. Kakkar AK, DeRuvo N, Chinswangwatanakul V, Tebbutt S, Williamson RC. Extrinsic-pathway activation in cancer with high factor VIIa and tissue factor. Lancet. 1995;346(8981):1004 1005.

5. Lijfering WM, Rosendaal FR, Cannegieter SC. Risk factors for venous thrombosis - current understanding from an epidemiological point of view. Br J Haematol. 2010;149(6): 824-833

6. Rosendaal FR. Venous thrombosis: a multicausal disease. Lancet. 1999;353(9159):11671173.

7. Drake TA, Cheng J, Chang A, Taylor FB, Jr. Expression of tissue factor, thrombomodulin, and E-selectin in baboons with lethal Escherichia coli sepsis. Am J Pathol. 1993;142(5):1458-1470.

8. Lupu C, Westmuckett AD, Peer G, et al. Tissue factor-dependent coagulation is preferentially up-regulated within arterial branching areas in a baboon model of Escherichia coli sepsis. Am J Pathol. 2005;167(4):1161-1172.

9. Song D, Ye X, Xu H, Liu SF. Activation of endothelial intrinsic NF-\{kappa\}B pathway impairs protein $\mathrm{C}$ anticoagulation mechanism and promotes coagulation in endotoxemic mice. Blood. 2009;114(12):2521-2529.

10. Pawlinski R, Mackman N. Cellular sources of tissue factor in endotoxemia and sepsis. Thromb Res. 2010;125 Suppl 1:S70-S73.

11. Posma JJ, Posthuma JJ, Spronk HM Coagulation and non-coagulation effects of thrombin. J Thromb Haemost. 2016;14(10):1908-1916.

12. Breitenstein A, Tanner FC, Luscher TF. Tissue factor and cardiovascular disease: quo vadis? Circ J. 2010;74(1):3-12

13. Gertz SD, Fallon JT, Gallo R, et al. Hirudin reduces tissue factor expression in neointima after balloon injury in rabbit femoral and porcine coronary arteries. Circulation. 1998;98(6):580-587.

14. Moons AH, Levi M, Peters RJ. Tissue factor and coronary artery disease. Cardiovasc Res. 2002;53(2):313-325.

15. Speidel CM, Eisenberg PR, Ruf W Edgington TS, Abendschein DR. Tissue factor mediates prolonged procoagulant activity on the luminal surface of balloon-injured aortas in rabbits. Circulation. 1995;92(11): 3323-3330.

16. van der Wal AC, Li X, de Boer OJ. Tissue factor expression in the morphologic spectrum of vulnerable atherosclerotic plaques. Semin Thromb Hemost. 2006;32(1):40-47.

17. Lopez-Pedrera C, Buendia P, Barbarroja N, Siendones E, Velasco F, Cuadrado MJ. Antiphospholipid-mediated thrombosis: interplay between anticardiolipin antibodies and vascular cells. Clin Appl Thromb Hemost. 2006;12(1):41-45.

18. Khechai F, Ollivier V, Bridey F, Amar M,
Hakim J, de Prost D. Effect of advanced glycation end product-modified albumin on tissue factor expression by monocytes. Role of oxidant stress and protein tyrosine kinase activation. Arterioscler Thromb Vasc Biol. 1997;17(11):2885-2890

19. Reverter JC, Tassies D, Font J, et al. Hypercoagulable state in patients with antiphospholipid syndrome is related to high induced tissue factor expression on monocytes and to low free protein s. Arterioscler Thromb Vasc Biol. 1996; 16(11):1319-1326.

20. Solovey A, Gui L, Key NS, Hebbel RP. Tissue factor expression by endothelial cells in sickle cell anemia. J Clin Invest. 1998;101 (9):1899-1904

21. Osterud B, Bjorklid E. Sources of tissue factor. Semin Thromb Hemost. 2006;32(1):1123.

22. Colucci M, Balconi G, Lorenzet $\mathrm{R}$, et al. Cultured human endothelial cells generate tissue factor in response to endotoxin. J Clin Invest. 1983;71(6):1893-1896.

23. Nawroth PP, Handley DA, Esmon CT, Stern DM. Interleukin 1 induces endothelial cell procoagulant while suppressing cell-surface anticoagulant activity. Proc Natl Acad Sci U S A. 1986;83(10):3460-3464.

24. Scarpati EM, Sadler JE. Regulation of endothelial cell coagulant properties. Modulation of tissue factor, plasminogen activator inhibitors, and thrombomodulin by phorbol 12-myristate 13-acetate and tumor necrosis factor. J Biol Chem. 1989;264(34):20705-20713.

25. Mackman N. Regulation of the tissue factor gene. FASEB J. 1995;9(10):883-889.

26. Mackman N, Morrissey JH, Fowler B, Edgington TS. Complete sequence of the human tissue factor gene, a highly regulated cellular receptor that initiates the coagulation protease cascade. Biochemistry. 1989;28(4):1755-1762.

27. Johannessen M, Moller S, Hansen T, Moens U, Van GM. The multifunctional roles of the four-and-a-half-LIM only protein FHL2. Cell Mol Life Sci. 2006;63(3):268-284

28. Kurakula K, Sommer D, Sokolovic M, et al. LIM-only protein FHL2 is a positive regulator of liver $\mathrm{X}$ receptors in smooth muscle cells involved in lipid homeostasis. Mol Cell Biol. 2015;35(1):52-62

29. Kurakula K, van der Wal E, Geerts D, van Tiel CM, de Vries CJ. FHL2 protein is a novel co-repressor of nuclear receptor Nur77. J Biol Chem. 2011;286(52):44336-44343.

30. Kurakula K, Vos M, Otermin Rubio I, et al. The LIM-only protein FHL2 reduces vascular lesion formation involving inhibition of proliferation and migration of smooth muscle cells. PLoS One. 2014;9(4):e94931.

31. Tran MK, Kurakula K, Koenis DS, de Vries CJ. Protein-protein interactions of the LIMonly protein FHL2 and functional implication of the interactions relevant in cardiovascular disease. Biochim Biophys Acta. 2016;1863(2):219-228.

32. Kuijpers MJ, de Witt S, Nergiz-Unal R, et al. Supporting roles of platelet thrombospondin-1 and CD36 in thrombus formation on collagen. Arterioscler Thromb Vasc Biol. 2014;34(6):1187-1192

33. de Vries CJ, van Achterberg TA, Horrevoets AJ, ten Cate JW, Pannekoek H. Differential display identification of 40 genes with altered expression in activated human smooth muscle cells. Local expression in atherosclerotic lesions of smags, smooth muscle activation-specific genes. I Biol
Chem. 2000;275(31):23939-23947.

34. Kurakula K, Vos M, van Eijk M, Smits HH, de Vries CJ. LIM-only protein FHL2 regulates experimental pulmonary Schistosoma mansoni egg granuloma formation. Eur J Immunol. 2015;45(11):3098-3106.

35. van den Hengel LG, Osanto S, Reitsma PH, Versteeg $\mathrm{HH}$. Murine tissue factor coagulant activity is critically dependent on the presence of an intact allosteric disulfide. Haematologica. 2013;98(1):153-158.

36. Mackman N, Fowler BJ, Edgington TS, Morrissey JH. Functional analysis of the human tissue factor promoter and induction by serum. Proc Natl Acad Sci U S A. 1990;87(6):2254-2258.

37. Germain M, Chasman DI, de Haan H, et al. Meta-analysis of 65,734 individuals identifies TSPAN15 and SLC44A2 as two suscep tibility loci for venous thromboembolism. Am J Hum Genet. 2015;96(4):532-542.

38. van Hylckama Vlieg A, Baglin CA, Luddington R, MacDonald S, Rosendaal FR, Baglin TP. The risk of a first and a recurrent venous thrombosis associated with an elevated D-dimer level and an elevated thrombin potential: results of the THE-VTE study. J Thromb Haemost. 2015; 13(9):1642-1652

39. Kuijpers MJ, Munnix IC, Cosemans JM, et al. Key role of platelet procoagulant activity in tissue factor-and collagen-dependent thrombus formation in arterioles and venules in vivo differential sensitivity to thrombin inhibition. Microcirculation. 2008;15(4):269-282

40. Wang L, Miller C, Swarthout RF, Rao M, Mackman N, Taubman MB. Vascular smooth muscle-derived tissue factor is criti$\mathrm{cal}$ for arterial thrombosis after ferric chloride-induced injury. Blood. 2009;113(3):705713.

41. Martorell L, Martinez-Gonzalez J, Rodriguez C, Gentile M, Calvayrac O, Badimon L. Thrombin and protease-activated receptors (PARs) in atherothrombosis. Thromb Haemost. 2008;99(2):305-315.

42. Oltrona L, Speidel CM, Recchia D, Wickline SA, Eisenberg PR, Abendschein DR. Inhibition of tissue factor-mediated coagulation markedly attenuates stenosis after balloon-induced arterial injury in minipigs. Circulation. 1997;96(2):646-652.

43. Guo Z, Zhang W, Xia G, et al. Sp1 upregulates the four and half lim 2 (FHL2) expression in gastrointestinal cancers through transcription regulation. Mol Carcinog. 2010;49(9):826-836

44. Dorfleutner A, Hintermann E, Tarui T, Takada Y, Ruf W. Cross-talk of integrin alpha3beta1 and tissue factor in cell migration. Mol Biol Cell. 2004;15(10):4416-4425.

45. Wixler V, Geerts D, Laplantine E, et al. The LIM-only protein DRAL/FHL2 binds to the cytoplasmic domain of several alpha and beta integrin chains and is recruited to adhesion complexes. J Biol Chem. 2000;275(43): 33669-33678.

46. Xia T, Levy L, Levillayer F, et al. The four and a half LIM-only protein 2 (FHL2) activates transforming growth factor $\beta$ (TGF- $\beta$ ) signaling by regulating ubiquitination of the E3 ligase Arkadia. J Biol Chem. 2013;288 (3):1785-1794.

47. Hayashi $\mathrm{H}$, Nakagami $\mathrm{H}$, Takami $\mathrm{Y}$, et al. FHL-2 suppresses VEGF-induced phosphatidylinositol 3-kinase/Akt activation via interaction with sphingosine kinase-1. Arterioscler Thromb Vasc Biol. 2009;29(6): 909-914 